Ann. Biol. anim. Bioch. Biophys., I967, 7 (3), 26r-279.

\title{
QUELQUES ASPECTS DU TRANSIT GASTRO-INTESTINAL CHEZ LE PORC
}

\author{
P. AUFFRAY, J. MARTINET, A. RERA'T \\ avec la collaboration technique de J.-C. Marcilloux \\ Station de Recherches sur l'Élevage des Porcs, \\ Laboratoire de Physiologie de la Lactation, \\ Centre national de Recherches zootechniques, 78 -Jouy-en-Josas \\ Institut national de la Recherche agronomique
}

\section{SOMMAIRE}

Le transit gastro-intestinal est étudié sous un aspect dynamique et quantitatif, à l'aide de la technique des fistules digestives permanentes. Simultanément, on enregistre la motricité du pylore et des régions gastriques et duodénales voisines au moyen de fins ballonnets placés dans les parois.

La méthode des fistules montre que la vidange stomacale est très précoce et très rapide si bien qu'au bout de 15 minutes, 30 à $40 \mathrm{p}$. I00 du volume de repas ingéré sont évacués dans le duodénum. L'arrivée d'une quantité aussi importante de bols alimentaires dans l'intestin entraîne alors une émission discontinue des effluents gastriques.

Ainsi, la vidange stomacale présente deux phases distinctes et ne peut s'exprimer sous la forme d'une courbe mathématique simple.

L'influence du volume et de la nature du régime sur l'évacuation gastrique a été envisagée. Le volume joue un rôle très important dans la régulation du transit gastro-intestinal et il existe une relation linéaire entre les volumes ingérés et les volumes évacués pendant la première phase du transit.

L'étude du fonctionnement du pylore a été abordée et les différents faits acquis tendent à montrer que cet organe jouerait un rôle secondaire dans le contrôle de la vidange stomacale. La régulation de cette dernière se ferait par des modifications de motricité au niveau du pylore et des différents segments gastriques.

Le $\mathrm{pH}$ des bols alimentaires ne semble pas occuper une place primordiale dans cette régulation.

\section{INTRODUCTION}

Le transit des aliments au niveau du tractus digestif a fait l'objet d'un grand nombre de travaux chez les espèces animales et chez 1'Homme. En ce qui concerne le Porc, le transit a surtout été envisagé sous un aspect global faisant appel à la technique des colorants ou à la méthode des abattages (RÉRAT et al., I963). Quant à la mesure du débit, au niveau d'un segment intestinal donné, il n'existe à notre 
connaissance que quelques études de CunNINGHAm (I962, I963) entreprises à l'aide de la technique des fistules digestives permanentes, technique déjà utilisée depuis plusieurs années chez le Mouton ou la Chèvre par PHI,LIPson et al. (I952), HogaN et al. (rg6o), Harris et al. (I962). Ajoutons que ces recherches de CunNingham étaient surtout envisagées sous un aspect biochimique et concernaient les parties postérieures de l'intestin grêle.

Actuellement, la technique des canules permanentes est la seule qui permette une étude du transit au niveau du tractus digestif, de repas riches en matières solides, sous un aspect dynamique et quantitatif. Aussi, l'avons-nous utilisée afin de connaître l'évolution de la vidange gastrique chez le Porc et de préciser certains aspects de sa régulation. Simultanément, nous avons enregistré la motilité gastrique afin de contrôler les perturbations possibles dues à la présence des canules.

\section{TECHNIQUES}

L'ensemble des recherches qui sont exposées ici ont été effectuées sur des porcs de race Large White pesant entre 50 et $70 \mathrm{~kg}$.

\section{A - Préparation chirurgicale des animaux}

a) Anesthésie.

Après un jeûne de 24 heures, les animaux reçoivent une injection intra-veineuse d'un mélange de Nembutal (éthyl-méthylbutylbarbiturate de sodium), de Penthiobarbital sodique (éthyl-méthylthiobarbiturate de sodium) et d'Atropine, dans les proportions qui suivent :

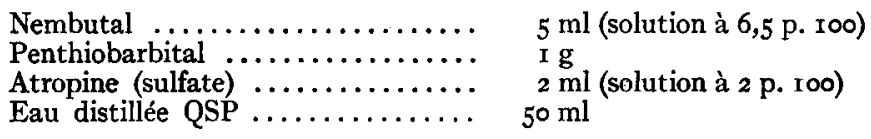

Après intubation trachéale, l'anesthésie est entretenue à l'aide d'un mélange gazeux (oxygèneprotoxyde d'azote), ce mélange pouvant ou non barboter dans du méthoxyfluorane (penthrane) suivant le degré d'anesthésie souhaitée.

\section{b) Mise en place des canules.}

On utilise des canules de polyvinyl dérivées du modèle de AsH (1962). Une canule destinée au prélèvement des effluents gastriques est placée au niveau de l'abouchement du canal pancréatique, ou à quelques centimètres en amont selon la longueur du duodénum.

Après incision de la paroi du duodénum, au niveau de la grande courbure, la canule est introduite dans la lumière intestinale, puis la fermeture de la plaie est réalisée à l'aide d'une suture d'enfouissement avec de la soie sertie $n^{0} o$. Une suture en bourse est réalisée autour de la canule avec de la soie sertie $n^{0}$ I. En une huitaine de jours, les tissus au niveau de la bourse sont nécrosés, ce qui entraîne des écoulements de contenus à l'extérieur des canules. Aussi, pour retarder l'apparition de ces fuites, une deuxième suture en bourse est réalisée autour des canules à l'aide du péritoine.

La canule traverse les parois du flanc droit immédiatement en arrière et en bas des dernières côtes. Elle est maintenue en place à l'aide de fils de nylon fixés dans une échancrure pratiquée à sa base. Ces fils sont glissés le long de la canule et noués sur un anneau de plastique. Ce mode de fixation empêche le retrait de la canule à l'intérieur de la cavité abdominale et le serrage de ces fils retarde l'apparition des fuites à l'extérieur. Cette fistule ainsi mise en place est dite fistule duodénale haute.

De cette manière, nous pouvons récolter tous les effluents gastriques, mais le prélèvement continu a pour effet d'accélérer la vidange stomacale. La réintroduction du chyme dans le duodénum est donc nécessaire : elle est réalisée à l'aide d'une deuxième canule placée une dizaine de centimètres en aval de la première. Toutefois, lors des réinjections, on assiste au reflux d'une certaine quantité 
d'aliment vers la première canule. Pour empêcher ce reflux, il est déconseillé d'utiliser un ballon placé entre les deux canules, car Phillipson (1952) a montré que la distension produite par son gonflement ralentit le transit. C'est pourquoi nous avons sectionné le duodénum entre les deux canules : les deux extrémités sont fermées á l'aide d'un surjet enfouissant, puis reliées entre elles par quelques points séparés pour éviter toute déchirure du mésentère ou du pancréas. Ĺes deux canules sont réunies à l'extérieur par un tube transparent. Leur diamètre intérieur est de $12 \mathrm{~mm}$.

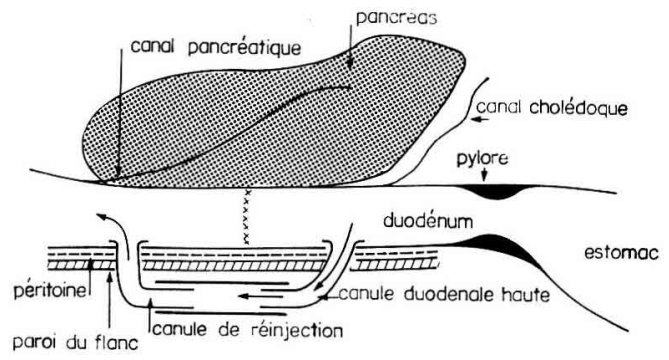

Schéma illustrant la mise en place des canules

La réalisation des bourses autour des canules doit être effectuée avec un soin méticuleux si l'on veut éviter les fuites le long de celles-ci. Il est ainsi possible d'utiliser des animaux pendant des périodes atteignant deux mois et cela malgré leur indocilité.

Sur le plan chirurgical, les difficultés concernent la brièveté du duodénum et du mésentère.

Afin de connaitre les perturbations possibles dues aux fistules, nous avons procédé à l'enregistrement de la motricité gastrique avant la mise en place des canules, pendant les manipulations et en dehors des prélèvements.

Cette étude est réalisée à l'aide de fins ballonnets de la grosseur d'un pois. Ils sont fabriqués de la manière suivante : l'extrémité d'un tube de polyéthylène très fin (diamètre intérieur : 4/10 mm) est chauffée à la flamme d'un bec Bunzen et fermée à l'aide d'une pince. A I ou 2 centimètres de cette extrémité, le tube est légèrement ramolli par chauffage puis dilaté par introduction d'air par l'extrémité ouverte du tube à l'aide d'une seringue munie d'une aiguille hypodermique. Avant leur mise en place, ces ballonnets sont remplis de liquide : l'extrémité du tube située du côté du ballonnet est sectionnée et on remplit le tube et le ballonnet de liquide de façon à chasser l'air. Puis on referme le tube à la flamme. Les ballonnets sont introduits dans les parois du bulbe duodénal, du pylore, des régions prépyloriques et antrales. Les tubes de polyéthylène sont fixés soigneusement au niveau du péritoine afin d'éviter une occlusion intestinale, puis après un long trajet sous la peau, ils sont extériorisés au niveau du dos. Au bout de quelques jours, une légère réaction tissulaire se produit autour des ballonnets qui les protège d'une dilatation excessive.

Pendant les manipulations, le tube rempli de liquide est relié à un capteur de pressions. L'enregistrement de la pression se fait sur un appareil à deux voies Sanborn.

L'estimation de la matière sèche se fait en prélevant une fraction des contenus stomacaux homogénéisés. Mais ces manipulations retardent la réintroduction des bols alimentaires dans le duodénum, ce qui entraîne une perturbation du transit.

\section{B - Protocole expérimental}

L'étude de la vidange stomacale est entreprise une huitaine de jours après l'opération. Les animaux sont alors placés dans une cage analogue à une cage à métabolisme, à laquelle ils s'adaptent très rapidement ( 3 à 4 jours). Ils reçoivent deux repas par jour, le premier à 8 heures et le second à 18 heures. Les manipulations se font généralement au cours du premier repas.

Les effluents stomacaux sont collectés dans un récipient en verre. Ce dernier est en relation avec un tube de verre fin qui sert d'indicateur de niveau. Le récipient collecteur est entouré d'un manchon permettant la circulation d'eau chaude destinée à maintenir les contenus à température constante. Les bols alimentaires sont réintroduits dans l'intestin à l'aide d'une pompe à galets munie d'un compte-tours.

L'étude du transit est effectuée généralement sur des périodes de 7 heures, chaque jour pendant plusieurs semaines.

Des prélèvements d'essai sont effectués pendant quelques jours jusqu'à reproductibilité des résultats. Pendant les manipulations, il faut veiller à ce que l'environnement du Porc soit toujours le même et surtout éviter tout bruit associé à la distribution de son régime. 
Porc $n^{\circ} 125$

Représentation du transit de 4 repas consécuti

\begin{tabular}{|c|c|c|c|c|c|c|c|c|c|c|c|c|}
\hline \multicolumn{2}{|c|}{$\begin{array}{l}\text { Temps depuis le début du repas } \\
\text { en quarts d'heure }\end{array}$} & 1 & 2 & 3 & 4 & 5 & 6 & 7 & 8 & 9 & 10 & 11 \\
\hline \multirow{4}{*}{$\begin{array}{l}\text { Quantités } \\
\text { émises } \\
\text { en ml }\end{array}$} & Repas $\mathrm{n}^{0} 1$. & 424 & 163 & 129 & 151 & 75 & 68 & 228 & 204 & 104 & 57 & 63 \\
\hline & Repas $n^{0} 2$. & 576 & 59 & 71 & 34 & 192 & 22 & 102 & 96 & 168 & $: 05$ & 56 \\
\hline & Repas $n^{\circ} 3 \ldots$ & 53 & 135 & 87 & 81 & 89 & 187 & 193 & 204 & 43 & 39 & 44 \\
\hline & Repas $n^{\circ} 4 \ldots$ & 558 & 234 & 76 & 111 & 106 & 54 & 58 & 159 & 262 & 50 & 30 \\
\hline
\end{tabular}

Porc $n^{\circ} 61014$

Transit de 3 repas contenant $600 \mathrm{~g}$

\begin{tabular}{|c|c|c|c|c|c|c|c|c|c|c|c|c|}
\hline \multicolumn{2}{|c|}{$\begin{array}{l}\text { Temps depuis le début du repas } \\
\text { en quarts d'heure }\end{array}$} & 1 & 2 & 3 & 4 & 5 & 6 & 7 & 8 & 9 & 10 & 11 \\
\hline \multirow{3}{*}{$\begin{array}{l}\text { Quantités } \\
\text { émises } \\
\text { en ml }\end{array}$} & Repas $n^{\circ} 1 \ldots$ & 600 & 70 & 95 & 535 & 500 & 355 & 215 & 115 & 115 & 175 & 115 \\
\hline & Repas $n^{\circ} 2 \ldots$ & 540 & 75 & 305 & 865 & 405 & 140 & 175 & 165 & 105 & 125 & 190 \\
\hline & Repas $n^{\circ} 3 \ldots$ & 485 & 275 & 315 & 155 & 800 & 140 & 85 & 180 & 55 & 65 & 260 \\
\hline
\end{tabular}

Porc $n^{\circ} 121$

Transit de 2 repas (moyenne) contenan

Temps depuis le début du repas en quarts d'heure

Quantités émises en $\mathrm{ml}$.

Porc $n^{\circ} 61639$

Transit de 4 repas contenant 700

\begin{tabular}{|c|c|c|c|c|c|c|c|c|c|c|c|c|}
\hline \multicolumn{2}{|c|}{$\begin{array}{l}\text { Temps depuis le début du repas } \\
\text { en quarts d'heure }\end{array}$} & \multirow{2}{*}{$\begin{array}{c}1 \\
900\end{array}$} & \multirow{2}{*}{$\begin{array}{l}2 \\
\\
31\end{array}$} & \multirow{2}{*}{$\begin{array}{c}3 \\
18\end{array}$} & \multirow{2}{*}{$\begin{array}{c}4 \\
12\end{array}$} & \multirow{2}{*}{$\begin{array}{c}5 \\
31\end{array}$} & \multirow{2}{*}{$\begin{array}{c}6 \\
25\end{array}$} & \multirow{2}{*}{$\begin{array}{c}7 \\
346\end{array}$} & \multirow{2}{*}{$\begin{array}{c}8 \\
403\end{array}$} & \multirow{2}{*}{$\begin{array}{r}9 \\
365\end{array}$} & \multirow{2}{*}{$\begin{array}{c}10 \\
170\end{array}$} & \multirow{2}{*}{$\begin{array}{r}11 \\
119\end{array}$} \\
\hline \multirow{4}{*}{$\begin{array}{l}\text { Quantités } \\
\text { émises } \\
\text { en ml }\end{array}$} & Repas no $1 \ldots \ldots \ldots$ & & & & & & & & & & & \\
\hline & Repas $n^{0} 2 \ldots$ & 1215 & 44 & 18 & 25 & 25 & 44 & 138 & 346 & 384 & 44 & 189 \\
\hline & Repas $n^{\circ} 2 \ldots$ & 743 & 12 & 189 & 611 & 434 & 384 & 258 & 151 & 302 & 100 & 75 \\
\hline & Repas $n^{\circ} 4 \ldots$ & 756 & 25 & 18 & 63 & 176 & 409 & 340 & 289 & 25 & 75 & 75 \\
\hline
\end{tabular}


U I

ntenant chacun $300 \mathrm{~g}$ de $\mathrm{M}$. S. et $900 \mathrm{ml} \mathrm{H}_{2} \mathrm{O}$ (Régime de base)

\begin{tabular}{r|r|r|r|r|r|r|r|r|r|r|r|r|r|r|r|r}
\hline \hline 12 & 13 & 14 & 15 & 16 & 17 & 18 & 19 & 20 & 21 & 22 & 23 & 24 & 25 & 26 & 27 & 28 \\
& & & & & & & & & & & & & & & & \\
\hline 238 & & & & & & & & & & & & & & & & \\
154 & 176 & 82 & 25 & 28 & 50 & 73 & 54 & 49 & 65 & 41 & 57 & 48 & 39 & 44 & 18 & 49 \\
228 & 155 & 71 & 91 & 98 & 113 & 64 & 39 & 116 & 73 & 53 & 52 & 74 & 75 & 27 & 45 & 78 \\
98 & 210 & 24 & 203 & 63 & 98 & 125 & 122 & 14 & 113 & 73 & 107 & 60 & 55 & 78 & 59 & 45 \\
\hline \hline
\end{tabular}

. S. et $1800 \mathrm{ml} \mathrm{H} \mathrm{H}_{2} \mathrm{O}$ (Régime de base)

\begin{tabular}{r|r|r|r|r|r|r|r|r}
\hline 12 & 13 & 14 & 15 & 16 & 17 & 18 & 19 & 20 \\
\hline 110 & 75 & 145 & 90 & 50 & 130 & 115 & 160 & 195 \\
305 & 125 & 185 & 90 & 95 & 200 & 340 & 180 & 120 \\
185 & 155 & 185 & 30 & 220 & 220 & 40 & 100 & 180 \\
\hline \hline
\end{tabular}

U 2

o g de M.S. $2100 \mathrm{ml} \mathrm{H}_{2} \mathrm{O}$ et (Régime de base)

\begin{tabular}{l|c|c|c|c|c|c|c|c|c|c|c|c|c|c|c|c}
\hline \hline 12 & 13 & 14 & 15 & 16 & 17 & 18 & 19 & 20 & 21 & 22 & 23 & 24 & 25 & 26 & 27 & 82 \\
\hline 130 & 109 & 75 & 205 & 207 & 142 & 162 & 237 & 177 & 155 & 200 & 162 & 90 & 80 & 185 & 150 & 75 \\
\hline
\end{tabular}

M. S. et $2100 \mathrm{ml} \mathrm{H} \mathrm{H}_{2} \mathrm{O}$ (Régime de base)

\begin{tabular}{r|r|r|r|r|r|r|r|r|r|r|r|r|r|r|r|r}
\hline \multicolumn{1}{r|r|r}{} & \multicolumn{1}{r|}{13} & 14 & 15 & 16 & 17 & 18 & 19 & 20 & 21 & 25 & 23 & 24 & 25 & 26 & 27 & 28 \\
\hline & & & & & & & & & & & & & & & & \\
\hline 56 & 69 & 37 & 220 & 207 & 69 & 195 & 37 & 151 & 151 & 170 & 118 & 100 & 163 & 75 & 107 & 113 \\
170 & 107 & 132 & 144 & 182 & 100 & 94 & 107 & 163 & 44 & 75 & 37 & 25 & 233 & 94 & 56 & 189 \\
245 & 138 & 201 & 88 & 283 & 63 & 107 & 201 & 182 & 144 & 37 & 138 & 189 & 63 & 144 & 176 & 132 \\
138 & 6 & 12 & 18 & 56 & 100 & 501 & 44 & 75 & 69 & 119 & 37 & 12 & 75 & 107 & 50 & 18 \\
\hline
\end{tabular}


Porc $n^{\circ} 323$

Transit de 7 repas contenant $600 \mathrm{~g}$

\begin{tabular}{|c|c|c|c|c|c|c|c|c|c|c|c|c|}
\hline \multicolumn{2}{|c|}{$\begin{array}{l}\text { Temps depuis le début du repas } \\
\text { en quarts d'heure }\end{array}$} & 1 & 2 & 3 & 4 & 5 & 6 & 7 & 8 & 9 & 10 & \\
\hline \multirow{7}{*}{$\begin{array}{l}\text { Quantités } \\
\text { émises } \\
\text { en ml }\end{array}$} & Repas no $1 \ldots$ & 635 & 75 & 40 & 500 & 430 & 235 & 190 & 70 & 175 & 115 & 60 \\
\hline & Repas $n^{\circ} 2 \ldots$ & 625 & 110 & 250 & 140 & 180 & 295 & 160 & 120 & 235 & 40 & 110 \\
\hline & Repas $n^{\circ} 3 \ldots$ & 535 & 130 & 420 & 390 & 90 & 405 & 260 & 240 & 120 & 105 & 190 \\
\hline & Repas $n^{\circ} 4 \ldots$. & 725 & 105 & 155 & 220 & 95 & 425 & 285 & 215 & 160 & 75 & 130 \\
\hline & Repas $n^{\circ} 5 \ldots$ & 570 & 70 & 245 & 145 & 200 & 540 & 225 & 200 & 240 & 150 & 135 \\
\hline & Repas $n^{\circ} 6 \ldots$ & 745 & 80 & 40 & 355 & 550 & 170 & 180 & 135 & 105 & 75 & 90 \\
\hline & Repas $n^{0} 7 \ldots \ldots \ldots$ & 725 & 105 & 60 & 185 & 290 & 275 & 160 & 195 & 125 & 110 & 155 \\
\hline
\end{tabular}

Porc $n^{\circ} 52007$

Transit de 2 repas contenant $700 \mathrm{~g}$ de $\mathrm{I}$

\begin{tabular}{|c|c|c|c|c|c|c|c|c|c|c|c|c|}
\hline \multicolumn{2}{|c|}{$\begin{array}{l}\text { Temps depuis le début du repas } \\
\text { en quarts d'heure }\end{array}$} & 1 & 2 & 3 & 4 & 5 & 6 & 7 & 8 & 9 & 10 & 11 \\
\hline Quantités & Repas no 1 & 1140 & 234 & 216 & 150 & 198 & 180 & 226 & 234 & 198 & 210 & 606 \\
\hline émises en $\mathrm{ml}$ & Repas $n^{\circ} 2 \ldots$ & 1092 & 210 & 162 & 300 & 108 & 216 & 126 & 18 & 282 & 318 & 462 \\
\hline
\end{tabular}

Porc $n^{0} 54262$

Transit de 2 repas chez un porc muni d'une fistule au niveau du car

Temps depuis le début du repas en quarts d'heure

Quantités émises par l'estomac en $\mathrm{ml}$. Quantités de bile émises en $\mathrm{ml} . . . \ldots .28$

\begin{tabular}{|r|r|r|r|r|r|r|r|r|r|r|}
\hline 1 & 2 & 3 & 4 & 5 & 6 & 7 & 8 & 9 & 10 & 11 \\
\hline- & & & & & & & & & & \\
\hline 580 & 40 & 105 & 120 & 125 & 30 & 25 & 60 & 365 & 240 & 210 \\
28 & 9 & 11 & 8 & 0 & 6 & 18 & 8 & 13 & 14 & 0 \\
\hline
\end{tabular}




\section{3}

S. (Régime de base) et $1800 \mathrm{ml} \mathrm{H} \mathrm{H}_{2} \mathrm{O}$

\begin{tabular}{l|r|r|r|r|r|r|r|r}
\hline 12 & 13 & 14 & 15 & 16 & 17 & 18 & 19 & 20 \\
& & & & & & & & \\
\hline 55 & 230 & 50 & 85 & 90 & 135 & 140 & 250 & 270 \\
90 & 95 & 70 & 175 & 365 & 20 & 110 & 300 & 185 \\
25 & 145 & 215 & 65 & 90 & 90 & 85 & 135 & 90 \\
10 & 170 & 210 & 180 & 145 & 40 & 210 & 120 & 95 \\
45 & 240 & 125 & 170 & 165 & 120 & 140 & 80 & 130 \\
29 & 130 & 50 & 100 & 40 & 220 & 75 & 100 & 65 \\
80 & 40 & 70 & 380 & 155 & 310 & 170 & 20 & 75 \\
\hline
\end{tabular}

(Régime de base) et $1800 \mathrm{ml} \mathrm{H}_{2} \mathrm{O}$

\begin{tabular}{|c|c|c|c|c|c|c|c|c|c|c|c|c|c|c|c|}
\hline 13 & 14 & 15 & 16 & 17 & 18 & 19 & 20 & 21 & 22 & 23 & 24 & 25 & 26 & 27 & 28 \\
\hline 378 & 234 & 138 & 234 & 294 & 312 & 450 & 468 & 252 & 342 & 360 & 480 & 162 & 30 & 250 & 18 \\
\hline 138 & 192 & 73 & 312 & 646 & 420 & 312 & 228 & 144 & 264 & 318 & 72 & 18 & 276 & 432 & 186 \\
\hline
\end{tabular}

U 4

lédoque et de fistules duodénales (repas $500 \mathrm{~g} \mathrm{M.S.} \mathrm{(Régime} \mathrm{de} \mathrm{base)} \mathrm{et} 1500 \mathrm{ml} \mathrm{H} \mathrm{H}_{2} \mathrm{O}$ )

\begin{tabular}{r|r|r|r|r|r|r|r|r|r|r|r|r|r|r|r|r}
\hline 2 & 13 & 14 & 15 & 16 & 17 & 18 & 19 & 20 & 21 & 22 & 23 & 24 & 25 & 26 & 27 & 28 \\
\hline 35 & 210 & 80 & 40 & 125 & 105 & 80 & 220 & 200 & 110 & 20 & 100 & 155 & 35 & 50 & 210 & 70 \\
15 & 17 & 24 & 12 & 16 & 11 & 13 & 15 & 80 & 18 & 17 & 23 & 17 & 21 & 11 & 24 & 21 \\
\hline
\end{tabular}


Le régime de base utilisé est un régime semi-synthétique formé de 65 p. 100 d'amidon, 7 p. 100 de caséine, 8 p. I0o d'huile d'arachide, $5 \mathrm{p}$. Ioo de cellulose et $5 \mathrm{p}$. 100 d'un mélange minéral et vitaminique. L'aliment ingéré est formé d'une partie de matière sèche pour deux ou trois parties d'eau.

D'une manière générale, le repas est consommé en trois à quatre minutes.

On mesure le $\mathrm{pH}$ sur les échantillons collectès à l'aide d'un pHmètre.

\section{RÉSULTATS}

Les résultats suivants sont tirés de l'observation expérimentale de 30 porcs fistulés et sur lesquels nous avons réalisé un total de 385 manipulations. Ces résultats reproduits dans les tableaux $I, 2,3,4$, ont été choisis aussi nombreux que possible en tenant compte surtout des variations individuelles et journalières.

\section{I - Aspect de la courbe de transit}

a) Repas normal.

En ordonnée, sont portées les quantités de contenus recueillies au niveau de la fistule duodénale haute exprimées en $\mathrm{ml}$, et en abscisse le temps divisé en quarts d'heure.

De l'examen de la courbe, et pour la période de temps considérée, il ressort qu'il existe deux phases dans la vidange gastrique : une phase initiale très rapide qui débute avant la fin de l'ingestion du repas et pendant laquelle les contenus évacués représentent 30 à $40 \mathrm{p}$. Ioo du volume ingéré, soit 20 à $25 \mathrm{p}$. Ioo pendant les cinq premières minutes (fig. I et 2). Ensuite apparaît une deuxième phase irrégulière avec émission discontinue, en jets, des bols alimentaires. Au cours de cette phase, les solides sont évacués d'une manière discontinue (fig. 3) ; leur réintroduction dans le duodénum entraîne un ralentissement, voire un blocage du transit gastro-intestinal. L'action ralentissante ou inhibitrice est d'autant plus importante que l'estomac présente un degré de vacuité plus marqué. L'apparition des solides au niveau de la fistule duodénale haute est toujours précédée de celle des liquides. Si on filtre sur gaze les effluents gastriques de telle sorte qu'on ne réintroduise que des liquides dans l'intestin, l'estomac n'évacue plus que des bols riches en solides.

Les volumes recueillis au bout de 7 heures sont environ deux fois plus élevés que les volumes ingérés. Ceci montre l'importance des sécrétions digestives.

b) Repas échelonnés.

Si le Porc consomme sa ration non plus en 3 à 4 minutes, mais d'une manière étalée sur $\mathrm{I}_{5}$ ou 30 minutes, on constate que l'évacuation gastrique reste toujours précoce et que les volumes recueillis pendant la phase initiale augmentent avec la durée d'ingestion (fig. 4).

\section{c) Composition des bols alimentaires.}

La quantité de matière sèche émise pendant le premier quart d'heure, exprimée en fonction de la quantité ingérée, représente ro à I $5 \mathrm{p}$. Ioo.

Le $\mathrm{pH}$ des échantillons prélevés reste constant pendant le premier quart d'heure et égal à celui de l'aliment utilisé $(6,3$ dans le cas de l'aliment standard à base d'amidon), puis devient alcalin et au bout de $3 / 4$ d'heure il baisse et oscille entre 5 et 3,5. 


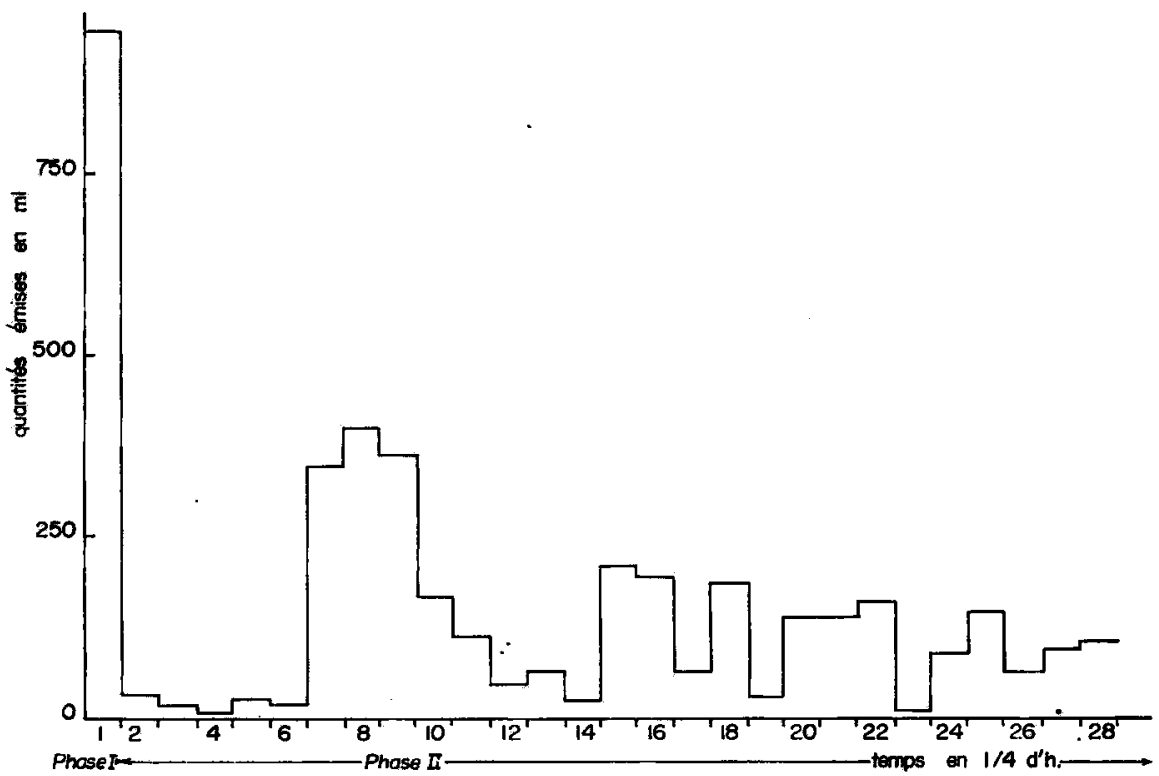

FIG. I. - Exemple de courbe montrant l'évolution du transit d'un repas standard au cours du temps

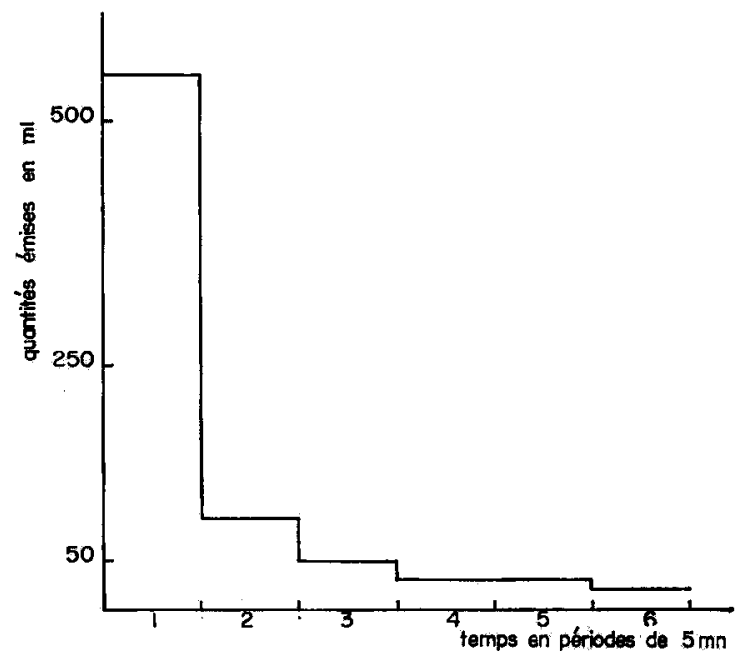

Fig. 2. - Evolution du transit pendant la première demi-heure (moyenne de 7 repas) 


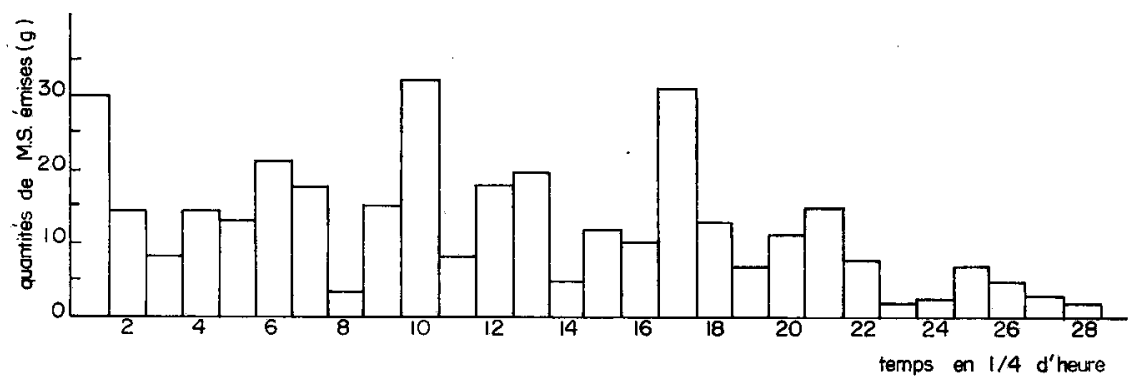

FIG. 3. - Courbe de vidange de la matière sèche

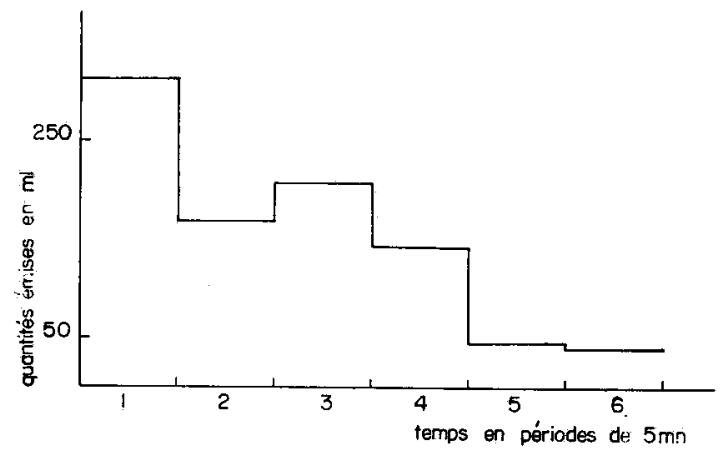

FIG. 4. - Vidange stomacale pendant la première demi-heure pour des repas consommés en un quart d'heure (moyenne de 3 repas)

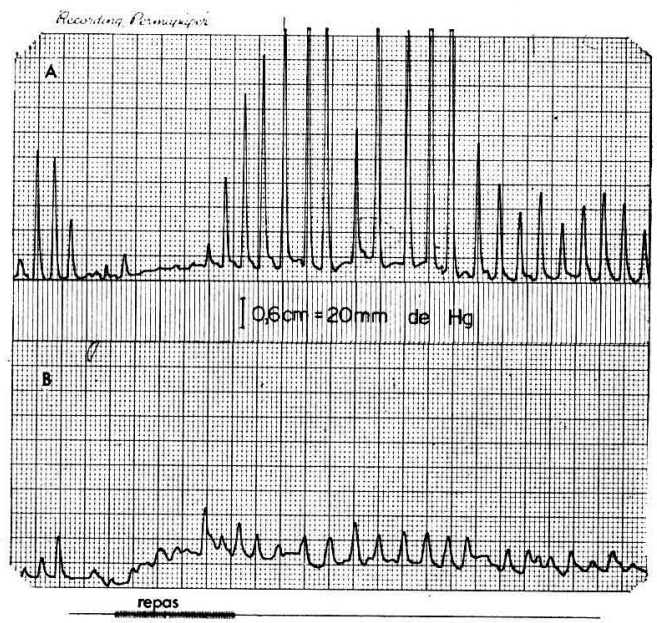

FIG. 5. - Enregistrement de la motricité : au niveau du pylore $(A)$, au niveau de la région prépylorique $(B)$. Vitesse de déroulement : $0,25 \mathrm{~mm} / \mathrm{s}$. 
d) Motricité.

Io Chez l'animal avec fistule et au cours des prélèvements. Pendant le repas, on observe une disparition de toute activité au niveau du pylore et ceci pendant une à deux minutes. Il s'agit d'un relâchement et non d'une contraction de fermeture du sphincter pylorique puisqu'une quantité importante d'aliment quitte l'estomac. Puis le pylore se contracte toutes les $15-20$ secondes et au même rythme que les régions prépyloriques et antrales (fig. 5). Ensuite apparaissent des phases de relâchement suivies de phases d'activité. Les inhibitions de la motricité accompagnent la réintroduction dans le duodénum de contenus à forte prédominance en matières solides (fig. 6).

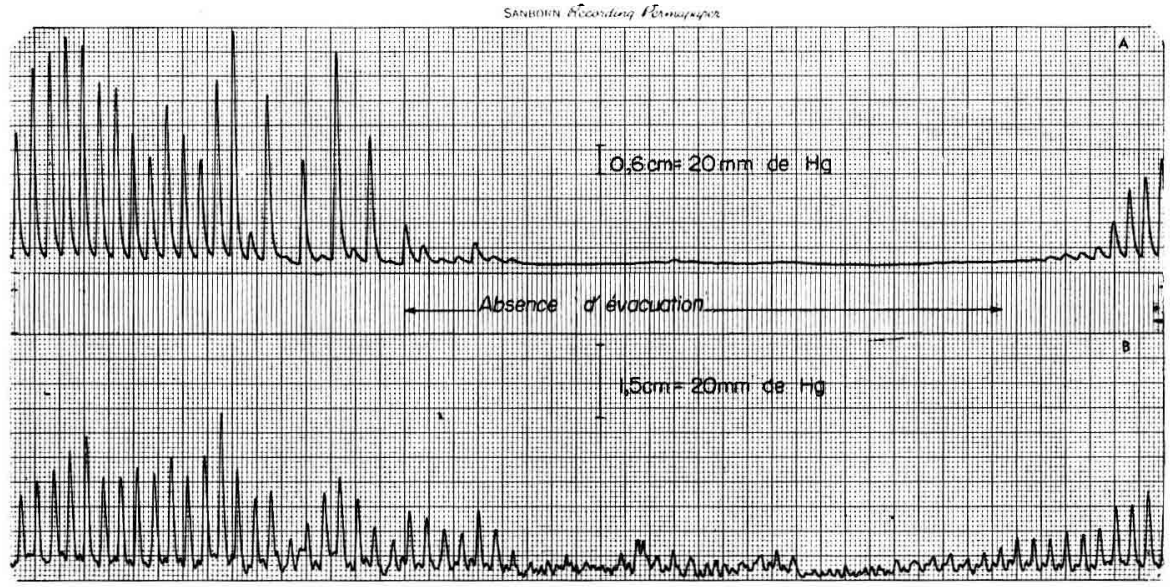

FIG. 6. - Enregistrement de la motricité dans le cas précis d partir de la quatrième heure, au niveau du pylore $(A)$, au niveau de la région antrale distale $(B)$. La véintroduction continue des effuents gastriques dans le duodénum entraîne d'abord une inhibition de la motricilé pylorique. La levée de cette inhibition se manifeste en premier lieu au niveau de l'antrum. Vitesse de déroulement $0,25 \mathrm{~mm} / \mathrm{s}$.

Au niveau des régions prépyloriques et antrales, au cours du repas, on enregistre une augmentation du tonus. Sur cette variation tonique se greffent des vagues de contraction de faible amplitude. Au niveau de ces régions, au cours de la deuxième phase de vidange gastrique, se produisent des alternances d'activité et de repos qui correspondent à celles des régions pyloriques. Toutefois, on peut observer des contractions au niveau de l'antrum alors que le pylore et les régions prépyloriques ne présentent aucune activité (fig. 7).

Dans tous les cas, les émissions de chyme stomacal résultent d'un fonctionnement unitaire de l'antrum, du pylore et du duodénum.

L'effet inhibiteur sur la motilité pylorique et gastrique des contenus solides réintroduits dans le duodénum dépend de leur volume, de leur nature et du degré de vacuité de l'estomac. De plus, cet effet est immédiat et très important lors de réinjections trop rapides.

$2^{\circ}$ L'animal sans fistule a une motricité similaire à la précédente. Toutefois, les variations cycliques au cours de la deuxième phase de vidange semblent moins marquées. 
$3^{\circ}$ L'animal avec fistule, mais chez lequel les effluents gastriques ne sont pas réinjectés, se distingue essentiellement des précédents par des vagues de contractions de grande amplitude et pratiquement ininterrompues.

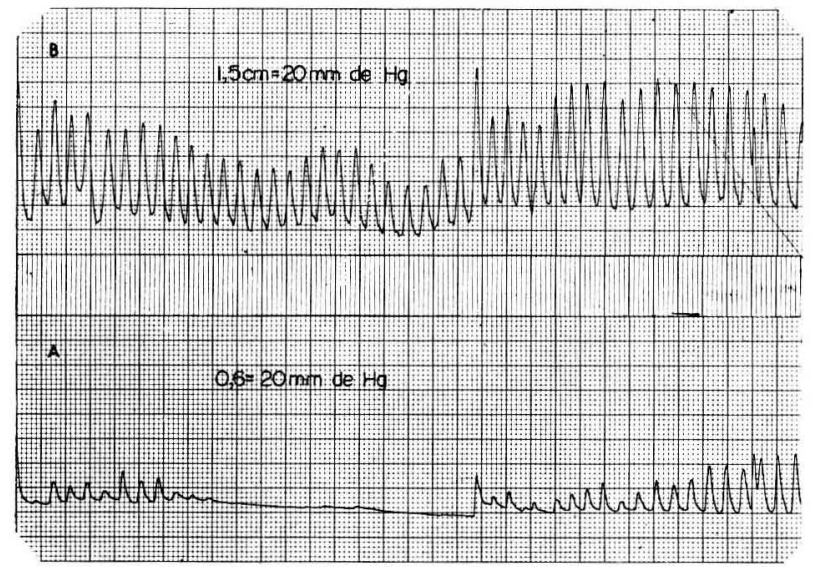

FIG. 7. - Enregistrement de la motricite dans le cas précis à partir de la cinquiène heure, au niveau de la région antrale proximale $(A)$, au niveau du pylore $(B)$. La réintroduction continue des bols alimentaires affecte très peu la motricité antrale. Vitesse de déroulement : $0,25 \mathrm{~mm} / \mathrm{s}$.

\section{II - Influence du volume ingéré et de la nature de l'aliment}

Io Influence du volume.

Plus le volume ingéré est important et plus grande sera l'évacuation gastrique. Entre le volume ingéré et le volume évacué pendant la première demi-heure, il existe une relation linéaire. L,es droites correspondantes (fig. 8) ne passent pas par

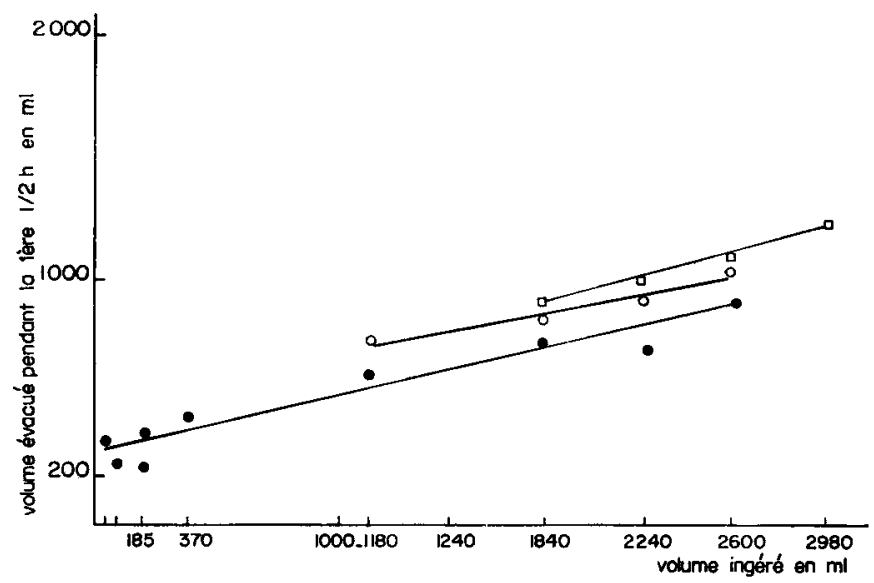

FiG. 8. - Relation entre le volume évacué pendant la première demi-heure et le volume ingéré

Porc no $54055 y=187+0,378 x, r=0,84$

Porc $n^{0} 54760 y=43^{8}+0,232 x, r=0,85$

Porc $\mathrm{n}^{\mathrm{a}} 3^{23} y=331+0,219 x, r=0,91$ 
l'origine car il y a toujours dans l'estomac des sécrétions et des reliquats des repas précédents, lesquels sont évacués en raison de l'excitation du Porc ayant reçu une ration insuffisante.

\section{$2^{0}$ Influence de la nature de l'aliment.}

Nous avons utilisé quelquefois des régimes naturels, mais plus souvent des régimes semi-synthétiques dont on peut aisément remplacer un constituant par un autre et dans les mêmes proportions. Dans un certain nombre d'essais, 1'amidon a été remplacé par du saccharose ou du glucose.

Pour un animal donné, nous obtenons une courbe caractéristique du transit, avec les deux phases précédemment décrites, sauf si les glucides sont sous forme de glucose ; dans ce cas, on observe une seule phase avec cependant une vidange toujours précoce des contenus stomacaux. Les courbes de transit se distinguent essentiellement entre elles par l'aspect des émissions cycliques des effluents gastriques (fig. 9).

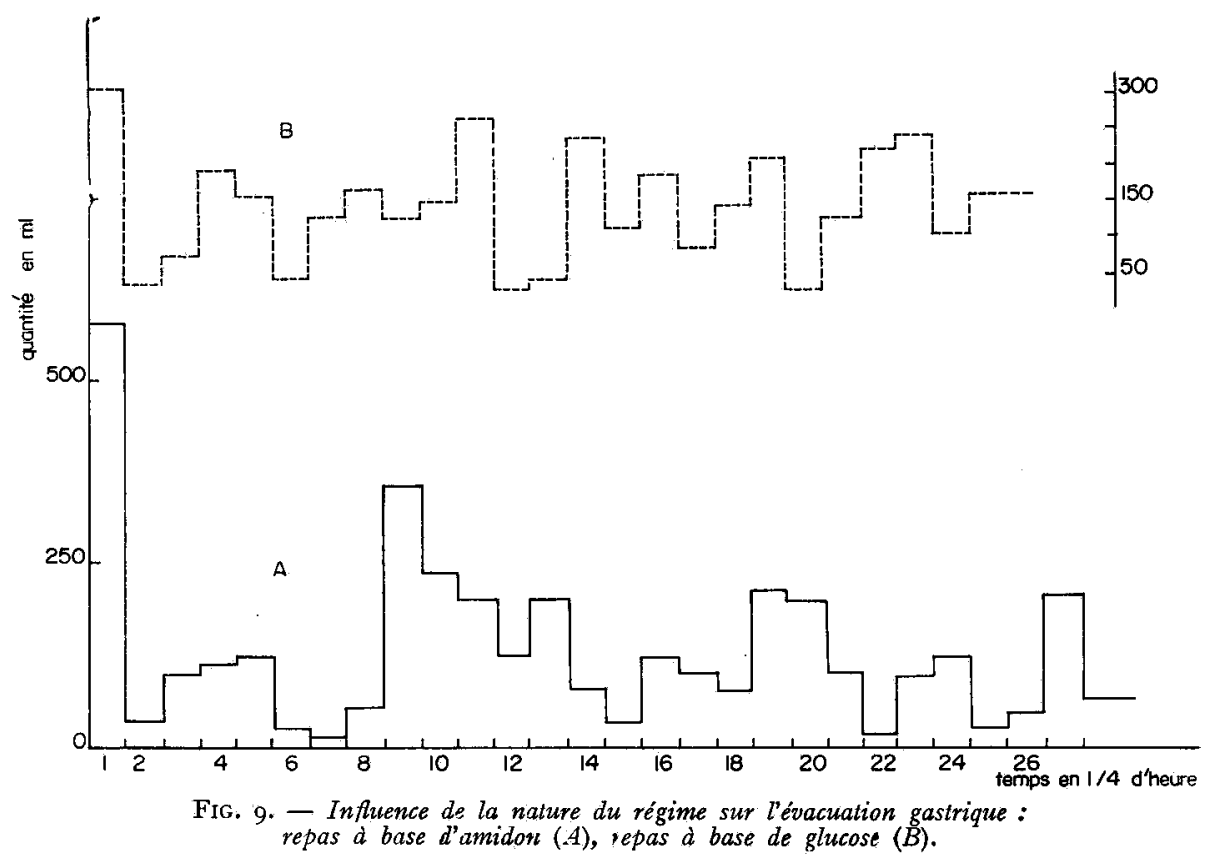

\section{III - Infuence $d u p H$}

Si nous remplaçons l'eau du repas par une solution tampon citrate $(\mathrm{pH}: 2,6)$ abaissant le $\mathrm{pH}$ de notre régime standard de 6,5 à 4 , on constate qu'il se produit une légère accélération du transit (fig. Io), mais en aucun cas nous n'observons de spasme du pylore lors de l'arrivée des bols alimentaires dans le duodénum bien que le $\mathrm{pH}$ de ces bols se maintienne entre 5 et 2 . Une solution d'acide chlorhydrique $\frac{\mathrm{N}}{20}$ $(\mathrm{pH}: 3,5)$ introduite dans le duodénum ne bloque pas la vidange stomacale pendant la phase initiale. 


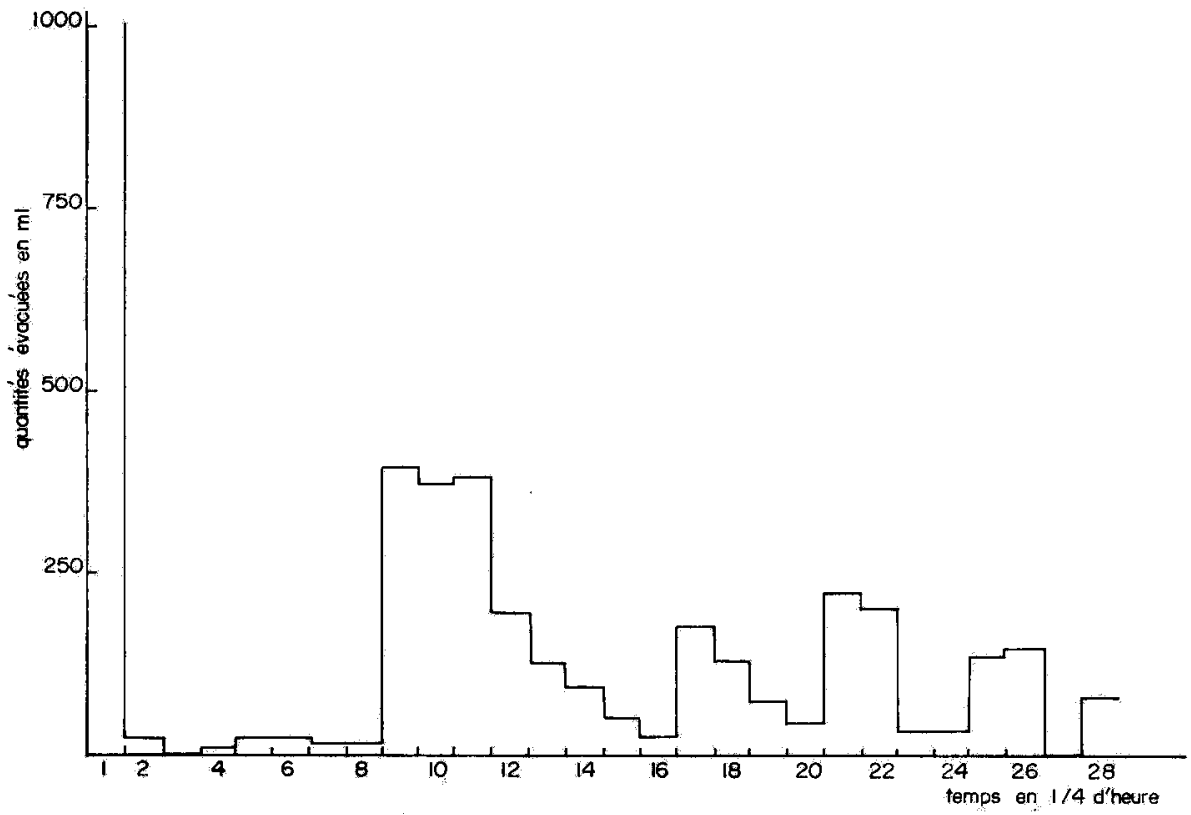

Fig. Io. - Évacuation d"un repas dont l'eau a été remplacte par une solution tampon citrate : $p H \quad 2,6$

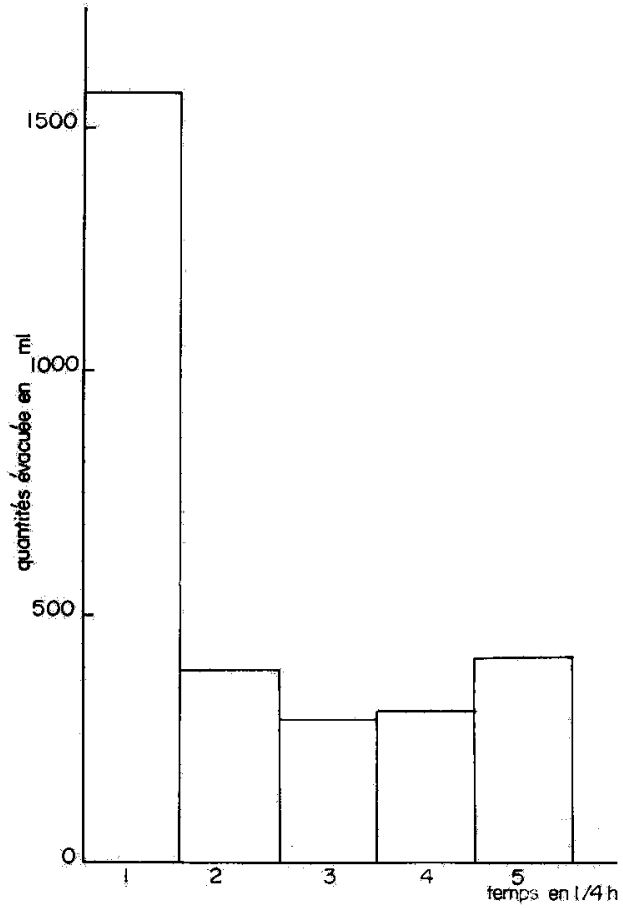

Fia. Ix. - Evacuation d'un repas constitué de $1850 \mathrm{ml}$ de lait écrémé 


\section{IV - Infuence de la consistance du régime sur la vidange gastrique}

$\mathrm{Si}$ on sépare les deux constituants du repas en fournissant d'abord la farine puis l'eau, on constate d'abord une évacuation des sécrétions et des résidus des repas précédents, puis des solides ingérés sous forme d'une pâte très consistante; mais dès que l'eau est ingérée, elle est évacuée très rapidement. De même, un repas liquide tel que le lait écrémé est évacué dans le duodénum en moins de 60 minutes (fig. II).

Il n'est pas possible en raison de la taille des canules d'utiliser des aliments grossiers.

\section{$\mathrm{V}$ - Infuence des aliments contenus dans l'intestin grêle sur la vidange gastrique}

Deux porcs munis de fistules duodénales sont utilisés simultanément. Après ingestion d'un repas, les effluents gastriques de l'un des porcs sont prélevés; ils sont réinjectés dans le duodénum du deuxième porc qui est alimenté à son tour. On constate alors, soit un ralentissement du transit, soit l'absence de toute évacuation. L'effet obtenu pour un volume donné de repas ingéré est d'autant plus marqué que la quantité de contenus réintroduite est plus importante et que ces contenus ont séjourné plus longtemps dans l'estomac.

\section{DISCUSSION}

Nous discuterons les différents résultats obtenus après les avoir rappelés brièvement.

\section{$\mathrm{A}-\mathrm{Du}$ point de vue méthodologique}

La difficulté principale rencontrée lors de l'utilisation des fistules permanentes réside dans 1'apparition de fuites autour des canules. Ces fuites provoquent une irritation cutanée rendant le porc agité et entraînent une vidange lente mais continue de l'estomac en dehors des prélèvements. Or, le transit d'un repas est influencé par les reliquats stomacaux du repas précédent.

La technique d'enregistrement de la motricité au moyen de fins ballonnets est très intéressante. Les ballonnets sont placés dans la paroi du tube digestif et non dans sa lumière évitant ainsi toute stimulation nerveuse ; de plus, les enregistrements obtenus sont très localisés.

Les enregistrements de la motricité avant et après la mise en place des canules sont sinilaires. Mais les variations journalières qui sont parfois importantes masquent les perturbations dues à la présence des fistules. Quant aux techniques de réinjection des contenus stomacaux dans le duodénum, elles sont susceptibles de modifier beaucoup l'allure du transit gastro-intestinal. Ces contenus doivent être réintroduits dans l'intestin au fur et à mesure de leur récolte et à la même vitesse. 


\section{B - Allure générale du transit}

Le transit gastro-intestinal n'est pas un phénomène continu puisque l'on observe sur la période de temps considéré deux phases bien nettes. HunT et McDonald (I954) qui, chez l'Homme, aspiraient à l'aide de sondes, les contenus stomacaux à différents intervalles de temps après ingestion de repas liquides, observaient également plusieurs phases dans la vidange gastrique. Cependant, HUNT et SPURRELI (I95I) concluaient à une allure exponentielle de la vidange stomacale. Toutefois, les résultats de ces auteurs ont été repris récemment dans leur laboratoire par Hopkins (I966) qui donne une nouvelle expression mathématique non exponentielle du transit gastrique.

Si on exprime les résultats sous forme de moyennes, on obtient des courbes d'évacuation relativement simples, mais qui ne correspondent pas au transit réel. En effet, il y a des variations individuelles et journalières importantes.

\section{C - Rôle du pylore et de la motricité}

L'introduction dans le duodénum d'aliments variés ou de chyme stomacal entraîne la disparition rapide de toute motilité du pylore, mais aussi de celle des régions prépyloriques et mêmes antrales. L'inhibition réflexe de la motricité à point de départ intestinal n'est donc pas limitée au pylore. De plus, cet effet est beaucoup plus difficile à obtenir lorsque l'estomac est en état de réplétion.

Ces faits sont en accord avec ceux de FRUMIN (I942) qui constata que 1'introduction dans le duodénum d'une solution d'acide chlorhydrique à o,5 p. Ioo inhibait les contractions gastriques. Des résultats similaires furent obtenus à l'aide de techniques différentes chez le Chien non anesthésié par Thomas (I93I), CRIDER et ThoMAS (r937), QUTGLEX et al. (I942).

Alvarez (I948), lors de nombreuses observations radiologiques, constatait parfois une absence de motricité au niveau du pylore et des régions prépyloriques alors que les contractions étaient bien marquées dans les autres territoires de l'estomac. Une compression gastrique était accompagnée d'un départ de chyme vers l'intestin.

Tous ces auteurs concluaient à un relâchement et non à une contraction du sphincter pylorique.

Ajoutons que dès I897, Von MERING constatait que la résection du pylore chez le Chien ne modifiait pas l'évacuation gastrique de liquides variés et que celle-ci se produisait toujours d'une manière intermittente. Par la suite, ceci s'est trouvé confirmé par de nombreux auteurs.

Nous avons vu que, chez le Porc, l'évacuation d'un repas était accompagnée de vagues de contractions au niveau du pylore et des régions gastriques voisines et également au niveau du bulbe duodénal. Toute dissociation dans cette coordination motrice entraîne une inhibition de la vidange de l'estomac. Toutefois, au début du repas, 1'estomac évacue environ $5 \mathrm{p}$. Ioo du volume ingéré sans que 1'on observe de motilité au niveau du pylore. Pendant cette période si l'amplitude des contractions est faible au niveau de l'estomac, par contre, il se produit une augmentation de tonus importante de la musculature stomacale. Il semblerait alors que la vidange stomacale se fasse en partie sous l'action d'une systole générale de l'estomac. 
Ce fonctionnement unitaire de l'antrum, du pylore et du duodénum a été observé depuis plusieurs années par de nombreux physiologistes et radiologistes. Ces constatations avaient conduit QUIGLEY et THOMAs à assimiler l'estomac et le duodénum à une pompe. Dans cette conception, les vagues de contraction péristaltiques provoquent des gradients de pression entre les deux organes, ce qui entraîne la vidange du chyme stomacal.

Nos études, qui confirment les faits apportés par d'autres auteurs, tendent à montrer que le pylore joue un rôle secondaire dans le contrôle de la vidange gastrique et que ce contrôle s'exercerait surtout par des modifications du péristaltisme gastroduodénal.

\section{$\mathrm{D}$ - Facteurs qui interviennent dans la régulation de la vidange gastrique}

Deux d'entre eux jouent un rôle important : ce sont le volume et la nature de l'aliment.

\section{a) Influence du volume et de la nature de l'aliment.}

Pendant la phase initiale, le passage de quantités importantes de contenus dans le duodénum entraîne un ralentissement de la vidange stomacale, mais le volume nécessaire pour provoquer ce ralentissement est variable. Il suffit de rappeler à cet égard l'influence du volume ingéré sur les quantités évacuées.

De plus, 1'introduction anticipée de nourriture dans 1'intestin grêle exerce une action plus ou moins importante sur l'estomac selon son volume, mais aussi selon le volume du repas ingéré. Pendant cette phase, la régulation de la vidange gastrique serait conditionnée essentiellement par des facteurs physiques, résultant du rapport entre les volumes présents dans l'estomac qui le stimulent et le volume des contenus dans l'intestin, lesquels provoquent sa distension mettant en jeu des mécano-récepteurs. Le freinage de l'évacuation stomacale par les contenus arrivant dans l'intestin se manifeste par une baisse progressive du tonus et de l'amplitude des contractions au niveau de l'estomac.

C'est lorsque l'équilibre est réalisé entre les effets produits au niveau de l'estomac et ceux produits au niveau de 1 'intestin que commence la deuxième phase de vidange.

L'importance du facteur volume est déjà une idée ancienne (L,UDIN, MORITZ, KATSCH, IgI2 ; CoHNhEIM et REYFus, I908) et a été bien développée par HuNT et SPURRELL (I95I).

Pendant la deuxième phase, les aliments vont subir un début de digestion dans l'estomac. Pendant cette action chimique se produisent des sécrétions importantes qui augmenteront le volume des contenus stomacaux. Simultanément, au niveau de 1 'intestin, se dérouleront des processus de digestion et d'absorption. On assistera alors à un déséquilibre en faveur de l'estomac entre l'effort propulsif développé à son niveau et les inhibitions d'origine intestinale. L'arrivée dans le duodénum d'un chyme stomacal, dont la constitution chimique dépend de la nature du repas ingéré, entraînera une nouvelle inhibition du transit gastro-intestinal. Ceci explique les émissions cycliques caractéristiques décrites au cours de la deuxième phase. 
b) Influence du $p H$.

Les différentes valeurs du $\mathrm{pH}$ au cours du transit d'un repas, 1'utilisation de repas tamponnés, tendent à prouver que le $\mathrm{pH}$ joue dans la vidange gastrique un rôle secondaire en regard des facteurs précédents. Ceci confirme une idée ancienne (LintvarefF, I903), et des auteurs comme Thomas chez le Chien (I940), Berk, Thomas et REYFus chez 1'Homme (I942) ont montré que l'acidité des contenus est rarement suffisante pour affecter la vidange stomacale. Rappelons que pendant la phase initiale, l'introduction dans le duodénum d'une quantité importante d'une solution d'acide chlorhydrique ne ralentit pas la vidange de l'estomac en état de réplétion.

En résumé, la vidange gastrique chez le Porc est très précoce et présente deux phases bien distinctes :

- Une phase initiale, pendant laquelle les aliments ne font que transiter au niveau de l'estomac.

Pendant cette phase ro à $\mathrm{I} 5 \mathrm{p}$. Ioo de la matière sèche ingérée pénètre dans l'intestin grêle ne subissant pas l'action du suc gastrique.

- Une deuxième phase avec émission discontinue des contenus stomacaux. La digestion se déroulera alors au niveau stomacal et au niveau intestinal.

Ainsi la vidange gastrique est discontinue et son expression ne saurait être assimilée à une courbe exponentielle.

Pendant cette phase, une quantité très importante de sécrétions est recueillie à la sortie de l'estomac. Dans les conditions physiologiques, le pylore semble jouer un rôle secondaire dans le contrôle de l'évacuation gastrique bien qu'il soit susceptible d'avoir son autonomie par rapport aux régions gastriques et duodénales voisines.

La régulation de la vidange stomacale par l'intestin se ferait essentiellement par les modifications de la motricité du pylore et des différents segments de l'estomac.

Loin de contrôler le transit gastro-intestinal, le $\mathrm{pH}$ n'interviendrait que pour une faible part dans sa régulation.

Reçu pour publication en mars 1967 .

\section{SUMMARY}

SOME ASPECTS OF GASTRO-INTESTINAL FLOW IN THE PIG

The dynamic and quantitative aspects of gastro-intestinal transport were studied by means of appropriate fistulas according to PHIL.LIPSON's technique (r952) adapted to the pig. Simultaneously, the motility of pylorus and adjacent parts of stomach and duodenum were studied by means of a special balloon technique. $70 \mathrm{~kg}$.

This investigation was carried out on 385 experiments on $3 \circ$ Large White pigs weighing 50 to

The fistula method showed that gastric emptying proceeds quickly at a high rate : within $15 \mathrm{mn}$, 30 to 40 per cent digesta had passed out to the duodenum. This considerable flow induced then an intermittent outpout of gastric effluents. Thus, gastric emptying shows 2 stages which cannot be reduced to an exponential function (fig. I).

These findings are not in accordance with those obtained with other species by means of other methods of studying gastro-intestinal flow (radiographic, slaughtering technique and aspiration of stomach contents at different lapses of time after the ingestion). 
The influences of the amount and composition of the diet were studied. A linear relationship exists between the ingested and emptied amounts during the first stage of the stomach emptying (fig. 8).

Pyloric mechanisms were investigated. During the stomach emptying, there was a single motive activity in the pylorus and adjacent muscles (antrum and duodenum). However, this coordination no longer exists during the meals, as the relaxation of pylorus shows it (fig. 5). During this time, 5 per cent only of the ingested volume evacuated the stomach. Any introduction of food or chyme into the duodenum induces an inhibitory response of the pylorus and adjacent muscles, even of the whole stomach (fig. 6 and 7). These facts incline us to the view that the pylorus only plays a secondary role in the regulation of gastric emptying. The prominent part would be played by alterations in motivity in the pyloric area and adjacent parts of the alimentary tract.

The $\mathrm{pH}$ of chyme does not seem to play a considerable part in the regulation.

\section{RÉFÉRENCES BIBLIOGRAPHIQUES}

Alvarez W. C., r948. An introduction to gastroenterology. Hoeber, N. Y.

Ash R. W., I962. Gastro-intestinal re-entrant cannulae for studies of digestion in sheep. Anim. Prod. 4, 309-3I 2 .

Berk J. E., Thomas J. E., Rehfuss M. E., i942. The reaction and neutralizing ability of the contents of the pyloric antrum and first part of the duodenum in normal dogs following an Ewald meal. $A m$. $J$. Physiol., 186, 157-166.

Cohnheim O., Dreyfus G. L., igo8. Zur physiologie und pathologie der Magenverdauung. Ztschr. Phwsiol. Chemie., 58, 50-83.

Crider J. O., Thomas J. E., I937. A study of gastric emptying with the pylorus open. Am. J. Dig. Dis., 4, 295-300.

Cunningham H. M., Friend D. W., Nicholson J. M. G., ig62. Note on a re-entrant fistula for digestion studies with pigs. Can. J. Anim. Sci., 42, 1 I 2-I 13.

Cunningham H. M., Friend D. W., Nicholson J. M. G., I963. Observations on digestion in the pig using a re-entrant intestinal fistula. Can. J. Anim. Sci., 43, 21 5-225.

Frumin Z. D., 1942. On the evacuation process of the stomach. Russian J. Physiol., Biol. Abstr., 16, 853.

Harris L. E., Phili ipson A. T., rg62. The measurement of the flow of food to the duodenum of sheep. Anim. Prod., 4, 97-1 16.

Hogan J. P., Phillipson A. T., 1960. The rate of flow of digesta and their removal along the digestive tract of sheep. Brit. J. Nutr., 14, 147-I56.

HoPKINS A., I 966 . The pattern of gastric emptying : a new view of old results. $J$. Physiol., 182, 144-I 49.

Hunt J. N., Spurrell W. R., 195I. Pattern of emptying of human stomach. J. Physiol., I13-157.

Hunt J. N., MAC Donald J., 1954. The influence of volume on gastric emptying. J. Physiol., 126, $459-474$.

KatsCH G., I9I2. Beitrag zum studium magenmotilität. Int. Beitr. Path. Ther. Ernähr. Stör., 8, 429-454.

LintVarefF S. I., Igo3. Ueber die Rolle der fette beim Utbergang des Mageninhalts in den Darm. Biochim. Zbl., 1, 96-97.

Mering J. Von., 1897. Zur Funktion des Magens. Verbandl. Kong. inn. Med., 15, 433-440.

Phillirson A. T., I952. The passage of digesta from the abomasum of sheep. J. Physiol., 116, 84.

Quigley J. P., Louckes H. S., 1962. Gastric emptying. Amer. J. Dig. Dis., 7, 672.

Rerat A., Lougnon J., 1963. Études sur le transit digestif chez le Porc. Ann. Biol. arim. Bioch. Biophys., 3, no hors-série, $\mathrm{I}, 2 \mathrm{I}-29$.

Thomas J. E., I931. The mechanism of gastric evacuation. J. Am. Med. Ass., 97, I663-1668.

Thomas J. F., 1940. The maximal acidity of the intestinal contents during digestion. Am. J. Dis., 7, I95-197.

Thomas J. E., I957. Mechanics and regulation of gastric emptying. Physiol. Rev., 37, 453. 